\title{
Dissipative dynamics in open XXZ Richardson-Gaudin models
}

\author{
Pieter W. Claeys $\odot^{*}$ and Austen Lamacraft $\odot$ \\ TCM Group, Cavendish Laboratory, University of Cambridge, Cambridge CB3 OHE, United Kingdom
}

(Received 15 September 2021; revised 9 December 2021; accepted 10 December 2021; published 14 January 2022)

\begin{abstract}
In specific open systems with collective dissipation the Liouvillian can be mapped to a non-Hermitian Hamiltonian. We here consider such a system where the Liouvillian is mapped to an XXZ Richardson-Gaudin integrable model and detail its exact Bethe ansatz solution. While no longer Hermitian, the Hamiltonian is pseudo-Hermitian $/ \mathcal{P} \mathcal{T}$ symmetric, and as the strength of the coupling to the environment is increased, the spectrum in a fixed symmetry sector changes from a broken pseudo-Hermitian phase with complex conjugate eigenvalues to a pseudo-Hermitian phase with real eigenvalues, passing through a series of exceptional points and associated dissipative quantum phase transitions. The homogeneous limit supports a nontrivial steady state, and away from this limit this state gives rise to a slow logarithmic growth of the decay rate (spectral gap) with system size. Using the exact solution, it is furthermore shown how at large coupling strengths the ratio of the imaginary to the real part of the eigenvalues becomes approximately quantized in the remaining symmetry sectors.
\end{abstract}

DOI: 10.1103/PhysRevResearch.4.013033

\section{INTRODUCTION}

No quantum system is truly isolated, and in the past decade there has been an increasing interest in the physics of open quantum many-body systems. The coupling to an external bath can give rise to gain and loss terms and decoherence, which can no longer be described by Hermitian dynamics, and open systems are generally described by a quantum master equation in terms of a non-Hermitian Liouvillian [1]. However, dissipation is not necessarily detrimental-the interplay between gain and loss can give rise to physics not accessible in closed systems and Hermitian dynamics. As one example, the system can exhibit $\mathcal{P} \mathcal{T}$ symmetry when the gain and loss terms are exactly balanced [2-5], and this symmetry can be spontaneously broken, leading to exceptional points and dissipative quantum phase transitions [6-8].

While it is generally impossible to exactly solve the master equation, exact solutions for specific integrable Liouvillians have started to appear in the literature. These range from noninteracting systems [9-16] to boundary-driven systems [17-22] and systems that can be mapped to a non-Hermitian Yang-Baxter integrable Hamiltonian [13,23-26]. A special class of the latter is those with collective dissipation [27-30], which will be the focus of this work. Specifically, we consider a Liouvillian that can be mapped to a Richardson-Gaudin integrable XXZ model. The integrability of this model was established by Rubio-García et al. [30], as a direct extension of earlier results of Ref. [27], and subsequently used to study

\footnotetext{
*pc652@cam.ac.uk

Published by the American Physical Society under the terms of the Creative Commons Attribution 4.0 International license. Further distribution of this work must maintain attribution to the author(s) and the published article's title, journal citation, and DOI.
}

the spectral statistics as an indicator of quantum chaos in open systems. The Hermitian Richardson-Gaudin XXZ model is known to exhibit a rich phase diagram [31-36], motivating a detailed study of the properties of the exact solution in the non-Hermitian case. As an additional motivation, the validity of approximate methods such as mean-field theory for open systems is still being studied [37-41], and exact solutions can serve as a useful benchmark for approximate numerical techniques.

In this work we detail the mapping to a non-Hermitian Hamiltonian and the subsequent exact solution of the model. This Hamiltonian, as well as the conserved charges, are shown to be pseudo-Hermitian, constraining the eigenvalues to be either real or part of a complex conjugate pair [2,42]. We show how eigenvalues change from complex conjugate pairs, when weakly coupled to the environment, to purely real eigenvalues when strongly coupled, after passing through exceptional points. At these exceptional points two eigenstates coalesce and the Liouvillian is no longer diagonalizable. Physically, these exceptional points correspond to dynamical dissipative phase transitions with nonanalytic relaxation rates throughout the spectrum, including for the leading decay mode [7].

All terms in the Lindblad equation and hence the Liouvillian can be chosen to be either homogeneous or inhomogeneous without losing the exact solvability and pseudo-Hermiticity. The homogeneous model admits a nontrivial steady state, and away from this limit we use the exact Bethe ansatz solution to show that in the inhomogeneous model the corresponding state decays with a small decay rate that scales logarithmically with system size. Here the exact Bethe ansatz solution is crucial in allowing us to obtain exact eigenvalues at system sizes out of reach of exact methods and establish this scaling. Somewhat surprisingly, in the purely dissipative regime close to the exceptional point the decay can be slower than in the weakly coupled regime. We additionally uncover a remarkable structure in the eigenspectrum, where at 
large coupling strengths the eigenvalues organize themselves on straight lines in the complex plane. The ratio of the imaginary to the real part of the eigenvalues becomes approximately quantized, indicating that the decay rate is proportional to oscillation frequency with a quantized proportionality factor.

This paper is organized as follows. In Sec. II we introduce the model and its exact Bethe ansatz solution through the mapping to a non-Hermitian integrable model. Section III discusses the spectrum of the model and its implications on the dynamics, with special attention paid to its symmetry properties and the homogeneous and strong-coupling limits, after which the commuting quantities associated with integrability are discussed in Sec. IV, as well as the numerical solution method for the Bethe equations. Section $\mathrm{V}$ is then reserved for conclusions.

\section{MODEL}

Assuming Markovian bath dynamics, the Lindblad equation [1] determines the time evolution of the density matrix $\rho$ as

$$
\dot{\rho}=-i[H, \rho]+\sum_{\alpha}\left[L_{\alpha} \rho L_{\alpha}^{\dagger}-\frac{1}{2} L_{\alpha}^{\dagger} L_{\alpha} \rho-\frac{1}{2} \rho L_{\alpha}^{\dagger} L_{\alpha}\right],
$$

where we set $\hbar=1$ and choose the system Hamiltonian $H$ and the jump operators $L_{\alpha}, \alpha \in\{+,-, z\}$ as

$$
\begin{aligned}
H & =\sum_{j}\left[\Omega+\omega_{j}\right] s_{j}^{z}, \\
L_{z} & =\sqrt{g_{0}} \sum_{j} s_{j}^{z}, \quad L_{ \pm}=\sqrt{g} \sum_{j} \sqrt{\omega_{j}} s_{j}^{ \pm} .
\end{aligned}
$$

The Hamiltonian describes a system of noninteracting spins, which we choose to have spin $1 / 2$, each subject to a local magnetic field. The \pm terms describe collective gain and loss, and the $z$ term describes a collective dephasing. The amplitudes $\omega_{j}$ in the Lindblad operators are related to the amplitudes in the Hamiltonian in order to obtain a solvable model, which can physically be achieved by introducing a detuning of the magnetic fields proportional to the terms in the jump operators. While the Hamiltonian itself is noninteracting, the collective Lindblad operators lead to an interacting Liouvillian. Furthermore, the gain and loss are balanced with equal strength $\mathrm{g}$, whereas the dephasing is tuned by an independent prefactor $g_{0}$.

As outlined in Ref. [27], the Lindblad operator can be mapped to a non-Hermitian Hamiltonian acting on a doubled Hilbert space. For example, a one-spin density matrix for spin $j$ can always be expanded as

$$
\rho_{j}=\frac{1}{2} \mathbb{1}_{j}+\sum_{\alpha} c_{j}^{\alpha} s_{j}^{\alpha},
$$

with $c_{j}^{\alpha}$ complex coefficients and $s_{j}^{\alpha}$ spin-1/2 operators, and the operators can be mapped to spin singlet and triplet states as

$$
\frac{1}{2} \mathbb{1}_{j} \rightarrow|0,0\rangle_{j}, \quad s_{j}^{z} \rightarrow|1,0\rangle_{j}, \quad s_{j}^{ \pm} \rightarrow \sqrt{2}|1, \pm 1\rangle_{j},
$$

such that each spin operator maps to a spin-1 state. The Liouvillian acts trivially on the singlet states, guaranteeing that the identity is always a trivial steady state, and under this mapping the action of Eq. (2) on the triplet states can be described by an operator [43]

$$
\begin{aligned}
\mathcal{L}= & i \sum_{j=1}^{L}\left[\Omega+\omega_{j}\right] \mathrm{S}_{j}^{z}-\mathrm{g}_{0} \sum_{j, k=1}^{L} \mathrm{~S}_{j}^{z} \mathrm{~S}_{k}^{z} \\
& -\mathrm{g} \sum_{j, k=1}^{L} \sqrt{\omega_{j} \omega_{k}}\left(\mathrm{~S}_{j}^{x} \mathrm{~S}_{k}^{x}+\mathrm{S}_{j}^{y} \mathrm{~S}_{k}^{y}\right),
\end{aligned}
$$

where the $\mathrm{S}^{\alpha}$ are now spin-1 operators. Here we use $L$ to denote the total number of triplet states, and take $\omega_{1} \ldots \omega_{L}$ to denote the corresponding amplitudes in the Liouvillian.

This operator is clearly no longer Hermitian, but can be interpreted as a Richardson-Gaudin model with factorizable interaction and complex interaction constant, which, however, does not preclude an exact solution. The (right) eigenstates are a direct generalization of the eigenstates for the Hermitian model (see Appendix A), and can be written as Bethe ansatz states of the form

$$
\left|v_{1} \ldots v_{N}\right\rangle=\prod_{a=1}^{N}\left(\sum_{j=1}^{L} \frac{\sqrt{\omega_{j}}}{\omega_{j}-v_{a}} \mathrm{~S}_{j}^{+}\right)|\emptyset\rangle,
$$

where $|\emptyset\rangle=|1,-1\rangle_{1} \otimes \cdots \otimes|1,-1\rangle_{L}$ is a vacuum state annihilated by all $\mathrm{S}_{j}^{-}$, and the wave function is parametrized by a set of (possibly complex) parameters $\left\{v_{1} \ldots v_{N}\right\}$, also known as rapidities, satisfying the Bethe equations

$$
-\frac{\mathrm{g}+i}{2 \mathrm{~g}}+\sum_{j=1}^{L} \frac{\omega_{j}}{\omega_{j}-v_{a}}-\sum_{b \neq a}^{N} \frac{v_{b}}{v_{b}-v_{a}}=0, \quad a=1 \ldots N .
$$

The Liouvillian has corresponding eigenvalues $\gamma\left(v_{1}, \ldots, v_{N}\right)$, with

$$
\begin{aligned}
\gamma\left(v_{1}, \ldots, v_{N}\right)= & (\mathrm{g}+i)\left(\sum_{a=1}^{N} v_{a}-\sum_{j=1}^{L} \omega_{j}\right) \\
& +i \Omega(N-L)-\mathrm{g}_{0}(N-L)^{2} .
\end{aligned}
$$

\section{SPECTRUM}

Before analyzing the eigenspectrum of the Liouvillian (5), it is useful to discuss its symmetries. First, it is clear that $\mathcal{L}$ conserves total spin- $z$ projection, i.e.,

$$
\left[\mathcal{L}, \mathrm{S}^{z}\right]=0, \quad \mathrm{~S}^{z}=\sum_{j} \mathrm{~S}_{j}^{z}
$$

Undoing the mapping from operators to states, acting with $\mathrm{S}^{z}$ on a state corresponds to commuting the operator with $\sum_{j} s_{j}^{z}$. All eigenstates of the Liouvillian are common eigenstates of $\mathrm{S}^{z}$, and it can easily be seen that

$$
\mathrm{S}^{z}\left|v_{1} \ldots v_{N}\right\rangle=(N-L)\left|v_{1} \ldots v_{N}\right\rangle .
$$

This implies that we can set $g_{0}=\Omega=0$ without loss of generality, since these simply correspond to a constant shift in the real and imaginary parts of the eigenvalues, respectively [see Eq. (8)]. This symmetry can also be observed from the Bethe equations (7), which are independent of both $g_{0}$ and $\Omega$. 
Second, and crucially, the Liouvillian is pseudo-Hermitian. Namely,

$$
\mathcal{L}^{\dagger}=P^{\dagger} \mathcal{L} P \quad \text { with } \quad P=\prod_{j=1}^{L} \mathrm{~S}_{j}^{x},
$$

which follows from the observation that the non-Hermitian part $i \sum_{j} \omega_{j} \mathbf{S}_{j}^{z}$ maps to $-i \sum_{j} \omega_{j} \mathbf{S}_{j}^{z}$ under Hermitian conjugation, which can be undone through the spin inversion operator $P$, whereas the remaining parts of the Liouvillian are both Hermitian and invariant under spin inversion.

This can also be interpreted as $\mathcal{P} \mathcal{T}$ symmetry, where a non-Hermitian Hamiltonian is invariant under a combined unitary (spin inversion) and antiunitary (complex conjugation) transformation, both of which square to identity [44,45]. On the level of the Liouvillian, spin inversion maps the Lindblad operator $L_{+}$to $L_{-}$and vice versa, exchanging the role of gain and loss. While the definition of $\mathcal{P} \mathcal{T}$ symmetry for a Liouvillian is more involved than that for a non-Hermitian Hamiltonian, as discussed in, e.g., Refs. [4,45], the mapping to a non-Hermitian model allows us to circumvent these subtleties.

The above identity (11) implies that left and right eigenstates are related through spin inversion. Furthermore, pseudo-Hermiticity implies that all eigenvalues either appear as part of a complex conjugate pair [2,42], in which case the two eigenstates are related through the corresponding $\mathcal{P} \mathcal{T}$ transformation, or as purely real, in which case the eigenstate is invariant under $\mathcal{P} \mathcal{T}$. The latter then correspond to states with unbroken $\mathcal{P} \mathcal{T}$ symmetry, while the former exhibit a broken $\mathcal{P} \mathcal{T}$ symmetry. Since $P^{\dagger} \mathrm{S}^{z} P=-\mathrm{S}^{z}$, only eigenstates with $\mathrm{S}^{z}=0$ (or $N=L$ ) can be invariant under $P$. In terms of operators, such states where $S^{z}=0$ correspond to the parts of the density matrix that preserve total spin $\sum_{j} s_{j}^{z}$ projection. This also implies that any eigenstate with nonzero $S^{z}$ will have a complex eigenvalue, and its complex conjugate corresponds to an eigenstate with $-\mathrm{S}^{z}$.

In Fig. 1, we plot the eigenspectrum for a small system of $L=3$ spins as $\mathrm{g}$ is increased from zero to some nonzero final value, focusing on the symmetry sector $S^{z}=0$. For concreteness, we consider a "picket-fence" model of evenly spaced levels $\omega_{i}=i, i=1 \ldots L$ [46], although all results in the following are independent of the specific model unless explicitly mentioned. For $\mathrm{g}=0$ the Lindblad operators vanish and the spectrum is purely imaginary, as expected, and all eigenvalues are either zero or part of a complex conjugate pair $\pm\left(E_{m}-E_{n}\right) i$, with $E_{m, n}$ eigenvalues of the noninteracting Hamiltonian $H$ from Eq. (2). The finite value of $\operatorname{Re}(\gamma) / \mathrm{g}$ as $\mathrm{g} \rightarrow 0$ in Fig. 1 indicates that the real part of the eigenvalues vanishes linearly with $\mathrm{g}$ for sufficiently small values of $\mathrm{g}$. As the coupling to the environment $g$ is turned on, the eigenvalues acquire a real part. Further increasing $\mathrm{g}$, the imaginary parts of the complex conjugate eigenvalues coalesce and vanish-the dynamics becoming purely dissipative once all eigenvalues have collapsed on the real line. At large $\mathrm{g}$, these purely real eigenvalues are then proportional to $\mathrm{g}$. Exceptional points occur both in the leading eigenvalue and throughout the spectrum, albeit at different values of $\mathrm{g}$, leading to nonanalyticities and hence transitions in the full Liouvillian spectrum as the coupling to the environment is varied.

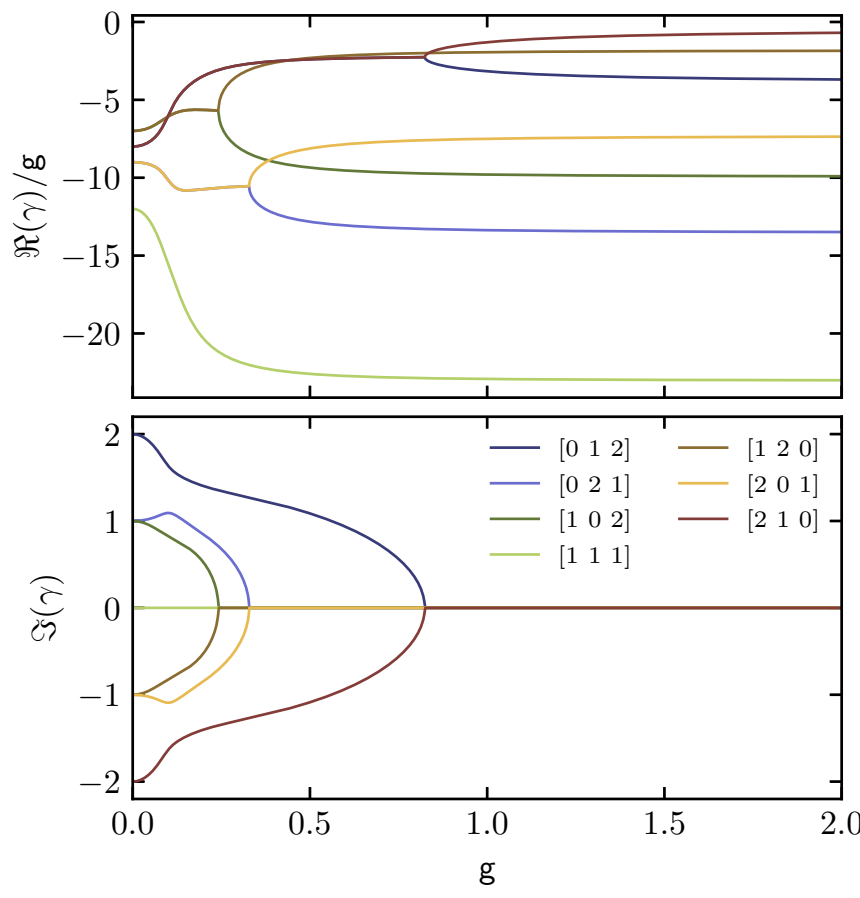

FIG. 1. Eigenspectrum for $L=N=3$ with $\omega_{i}=i, i=1 \ldots L$ as $\mathrm{g}$ is varied. Note that the real part is rescaled by $\mathrm{g}$, such that a finite value of $\operatorname{Re}(\gamma) / \mathrm{g}$ as $\mathrm{g} \rightarrow 0$ indicates purely imaginary eigenvalues in this limit. The eigenstates are labeled by the spin occupation numbers $\left[n_{1} n_{2} n_{3}\right]$ in the noninteracting limit $\mathrm{g}=0$, with $N=\sum_{j} n_{j}$.

This behavior readily extends to larger system sizes. In Fig. 2 we plot the full spectrum for a system of $L=8$ spins at two different values of the coupling strength $\mathrm{g}$. The spectrum is symmetric with respect to the real axis because of the pseudo-Hermiticity, and symmetric states have opposite values of $\mathbf{S}^{z}$. Different limiting behaviors can be observed: at small $\mathrm{g}$ almost all states correspond to complex conjugate pairs, whereas for larger $g$ the vast majority of states with $\mathrm{S}^{z}=0$ have collapsed onto the real axis. If we would further increase $\mathrm{g}$ all states in this symmetry sector eventually become real. In both limits there is a nonvanishing spectral gap and a "continuum" of nearby states. However, for small g this gap is determined by a pair of complex conjugate eigenstates, whereas at large $g$ the spectral gap is determined by a single real and nondegenerate eigenvalue. While the real part of most states is proportional to $g$, the spectral gap in fact decreases after passing through an exceptional point (as can also be observed in Fig. 1).

In terms of the dynamics, a smaller spectral gap corresponds to a slower decay rate. For small $g$ the leading decay mode will exhibit oscillations with a frequency set by the imaginary part of the leading eigenvalues, whereas for larger $\mathrm{g}$ the leading mode is purely dissipative but (possibly) decaying at a slower rate. Exactly at the exceptional point where the complex conjugate eigenvalues coalesce, the Liouvillian is no longer diagonalizable and a two-dimensional Jordan block will appear in its Jordan block decomposition, leading to critical dynamics $t e^{-|\gamma| t}$ with $\gamma$ the leading eigenvalue [7]. The exceptional point is accompanied by a nonanalytic behavior of this leading eigenvalue, leading to a dissipative quantum 

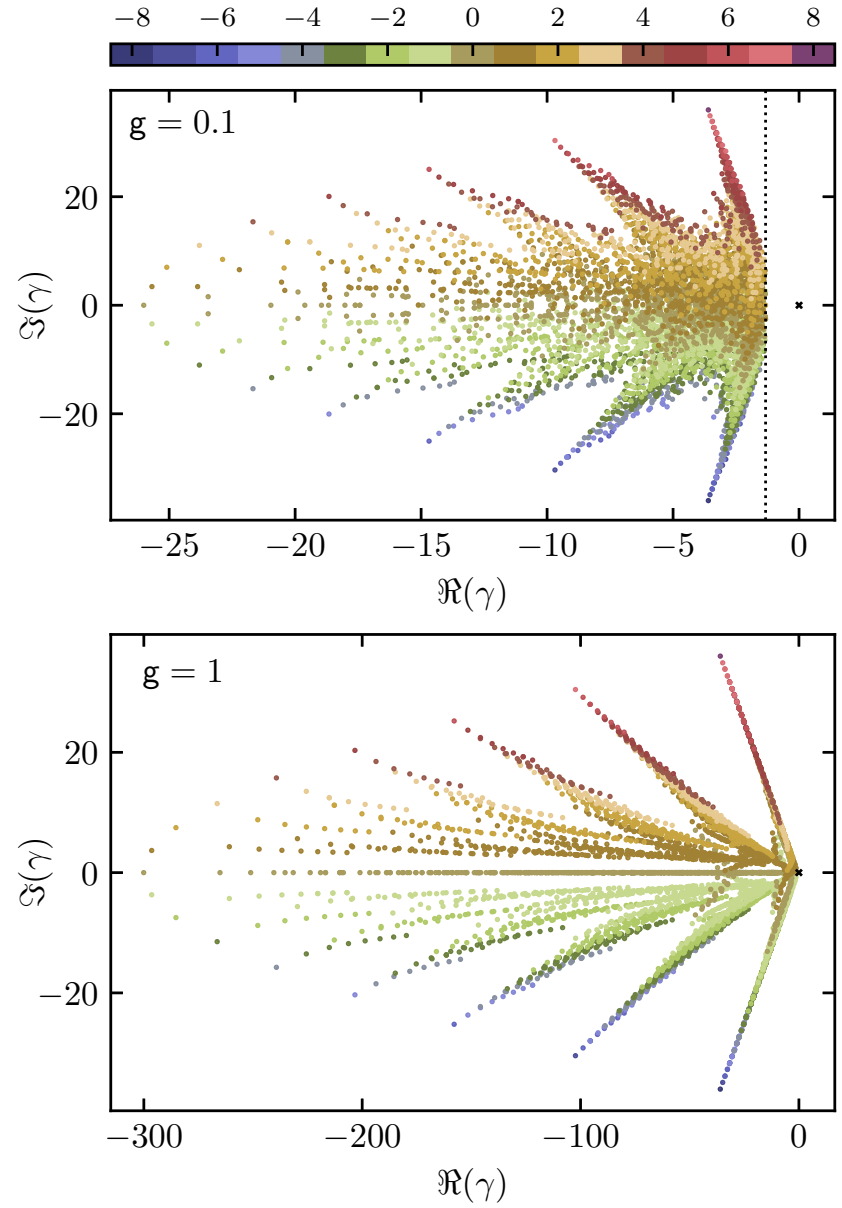

FIG. 2. Eigenspectrum for $\omega_{i}=i, \quad i=1 \ldots L$ for fixed $\mathrm{g}$ and $L=8$. The eigenstates are color coded according to $\mathrm{S}^{z}$. For $\mathrm{g}=0.1$ the dotted vertical line indicates the spectral gap $\approx-1.328$ corresponding to a pair of complex conjugate states, for $g=1$ the spectral gap $\approx-0.674$, and the cross indicates the origin as reference.

phase transition. This transition can also be interpreted as the spontaneous breaking of $\mathcal{P} \mathcal{T}$-symmetry/pseudo-Hermiticity, since for large $g$ the leading mode is nondegenerate and hence necessarily invariant under $\mathcal{P} \mathcal{T}$ symmetry, whereas at small $\mathrm{g}$ the two leading modes are no longer invariant under $\mathcal{P} \mathcal{T}$ symmetry, but rather get mapped to each other under the combination of spin inversion and complex conjugation.

This behavior is even more pronounced for nonzero $\mathrm{g}_{0}$. In this case all eigenvalues acquire an additional shift of the real value $-\mathrm{g}_{0}\left(\mathrm{~S}^{z}\right)^{2}=-\mathrm{g}(N-L)^{2}$, such that the decay rate of all sectors with nonzero $S^{z}$ is increased. Only the relevant $S^{z}=0$ sector, in which the transition occurs, is left invariant by both a nonzero $g_{0}$ and nonzero $\Omega$. The latter induces an additional shift in the complex value of all sectors with nonzero $S^{z}$, leading to global shift in all oscillation frequencies. As such, while the transition only occurs in the symmetry sector $\mathrm{S}^{z}=$ 0 , this sector generally contains the leading eigenvalue and for sufficiently large $g_{0}$ all dynamics are determined purely by this sector, with all other sectors rapidly decaying. The other symmetry sectors still exhibit nontrivial behavior as $g$ is increased, since it can be seen that these eigenvalues organize themselves on (approximately) straight lines in the spectrum

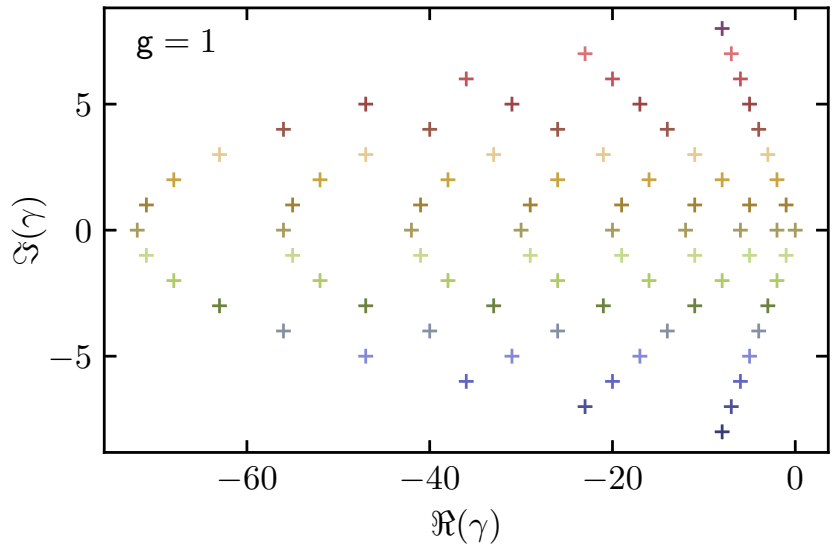

FIG. 3. Eigenspectrum for the homogeneous model with $\omega_{j}=$ $\omega=1, \forall j$ for fixed $g$ and $L=8$. The eigenstates are color coded according to $M$, as in Fig. 2.

where $\operatorname{Im}(\omega) \propto \operatorname{Re}(\omega)$. These lines will be discussed in more detail in Sec. III B. In the following, we will consider different limits of this model where the exact solution allows for additional insight.

\section{A. Homogeneous limit}

One limit where the exact solution is particularly simple is the limit where all $\omega_{j}$ are equal, i.e., $\omega_{j}=\omega, \forall j$. In this case the model can be recast in terms of total spin operators $\mathrm{S}_{\mathrm{tot}}^{\alpha}=\sum_{j} \mathrm{~S}_{j}^{\alpha}$ as

$$
\begin{aligned}
\mathcal{L} & =i(\Omega+\omega) \mathrm{S}_{\text {tot }}^{z}-\mathrm{g}_{0}\left(\mathrm{~S}_{\mathrm{tot}}^{z}\right)^{2}-\mathrm{g} \omega\left(\mathrm{S}_{\mathrm{tot}}^{x} \mathrm{~S}_{\mathrm{tot}}^{x}+\mathrm{S}_{\mathrm{tot}}^{y} \mathrm{~S}_{\mathrm{tot}}^{y}\right) \\
& =i(\Omega+\omega) \mathrm{S}_{\mathrm{tot}}^{z}-\mathrm{g}_{0}\left(\mathrm{~S}_{\mathrm{tot}}^{z}\right)^{2}-\mathrm{g} \omega\left[\left(\overrightarrow{\mathrm{S}}_{\mathrm{tot}}\right)^{2}-\left(\mathrm{S}^{z}\right)^{2}\right] .
\end{aligned}
$$

The eigenstates immediately follow as degenerate multiplets expressed in $\left|\mathrm{S}_{\text {tot }}, \mathrm{S}_{\text {tot }}^{z}\right\rangle=|S, M\rangle$, with total spin $S$ and total spin projection $M$. The corresponding eigenvalues $\gamma_{S, M}$ are given by

$$
\gamma_{S, M}=i \omega M-g \omega\left[S(S+1)-M^{2}\right],
$$

again setting $\mathrm{g}_{0}=\Omega=0$ for convenience. In fact, the same model is obtained as for the homogeneous limit of the XXX Richardson-Gaudin model studied in Ref. [27], only now with an interaction constant $\mathrm{g} \omega$ instead of $\mathrm{g}$. The degeneracy of these eigenvalues follows from the total number of ways in which $L$ spin-one particles can be coupled to total spin $S$, as given by the Riordan numbers $[27,47,48]$.

The spectrum is shown in Fig. 3 for a system with $L=8$ spins. The homogeneous model now has a nontrivial steady state within the triplet sector given by the state $S_{\text {tot }}=0$, i.e., the fully isotropic state (see also Ref. [27]). The real part is maximized when $M= \pm S$, and the leading eigenvalues are a pair of complex conjugate eigenvalues with $S=|M|=1$, resulting in a spectral gap $g \omega$. Furthermore, all states with $\mathrm{S}^{z}=M=0$ are purely real at all values of $\mathrm{g}$, such that no transition occurs as $\mathrm{g}$ is varied. In the $\mathrm{S}^{z}=0$ sector of the homogeneous model, pseudo-Hermiticity is unbroken at all coupling strengths. The nontrivial steady state and this lack of a transition is particular to the homogeneous model. 
More generally, note that the ratio of the real and imaginary parts satisfies

$$
g \frac{\operatorname{Im}\left(\gamma_{S, M}\right)}{\operatorname{Re}\left(\gamma_{S, M}\right)}=-\frac{M}{S(S+1)-M^{2}},
$$

independent of $\omega$. The real part is proportional to $\mathrm{g}$, whereas the imaginary part is independent of $\mathrm{g}$, reminiscent of Fig. 2. Plugging in $M=\mp S$ returns a ratio \pm 1 , plugging in $M=$ $\mp(S-1)$ returns a ratio $\pm(S-1) /(3 S-1) \approx \pm 1 / 3$ in the limit of large $S$, etc. This is already indicative of the lines observed in the bottom panel of Fig. 2, which will be shown to be smoothly connected to the solutions with fixed $S-M$.

\section{B. Strong-coupling limit}

Part of the structure of the homogeneous model is recovered in the strong-coupling limit where $g$ is sufficiently large. The clearest connection is that all eigenvalues for $\mathrm{S}^{z}=0$ collapse to the real line, resulting in an unbroken pseudoHermitian phase within this symmetry sector. In this limit we can also treat the non-Hermitian contribution to $\mathcal{L}$ as a perturbation on top of the Hermitian interaction part. While there is no longer a nontrivial steady state, away from the homogeneous limit we can consider the behavior of the leading eigenvalue. For $g$ sufficiently small we observed that the leading eigenvalue belongs to a pair of complex conjugate states, whereas for $\mathrm{g}$ sufficiently large the leading eigenvalue is purely real and nondegenerate. As one particular application, in the latter limit the leading mode can be considered a perturbative correction of the ground state of the related Hermitian Hamiltonian

$$
H=\sum_{j=1}^{L} \omega_{j} \mathrm{~S}_{j}^{z}+\mathrm{g} \sum_{j, k=1}^{L} \sqrt{\omega_{j} \omega_{k}}\left(\mathbf{S}_{j}^{x} \mathbf{S}_{k}^{x}+\mathrm{S}_{j}^{y} \mathbf{S}_{k}^{y}\right) .
$$

In the homogeneous limit, the ground state of this model is clearly given by the nontrivial steady state with $S_{\text {tot }}=0$, and we can now check what happens to this state in the inhomogeneous picket-fence model.

In order for the Bethe approach to be advantageous, we need to be able to explicitly target states of interest, e.g., the leading decay mode. However, the leading mode at (very) small coupling is not adiabatically connected to the leading mode at strong coupling, as also clear from Fig. 1. This is consistent with the Hermitian model, which undergoes a phase transition at $g=1$. However, we observe that the ground state at large coupling in the Hermitian model is adiabatically connected to the leading mode of the non-Hermitian model at strong coupling. Empirically, we find that this ground state is connected to the state $\left(\mathrm{S}_{1}^{+}\right)^{2}\left(\mathrm{~S}_{3}^{+}\right)^{2}\left(\mathrm{~S}_{5}^{+}\right)^{2} \ldots|\emptyset\rangle$ as $\mathrm{g} \rightarrow 0$ and to a nontrivial steady state with $S_{\text {tot }}=0$ in the homogeneous limit, allowing us to explicitly find the spectral gap without having to iterate over all Bethe states.

Using this correspondence, we compare the scaling with system size of the real part of the leading eigenvalue for the picket-fence model at two values of $g$ in Fig. 4. We have chosen $\mathrm{g}=0.25$ to be small enough that the leading eigenvalue is still part of a complex conjugate pair at all considered system sizes, but large enough that we are away from the weak-coupling limit in which the eigenvalues can be treated

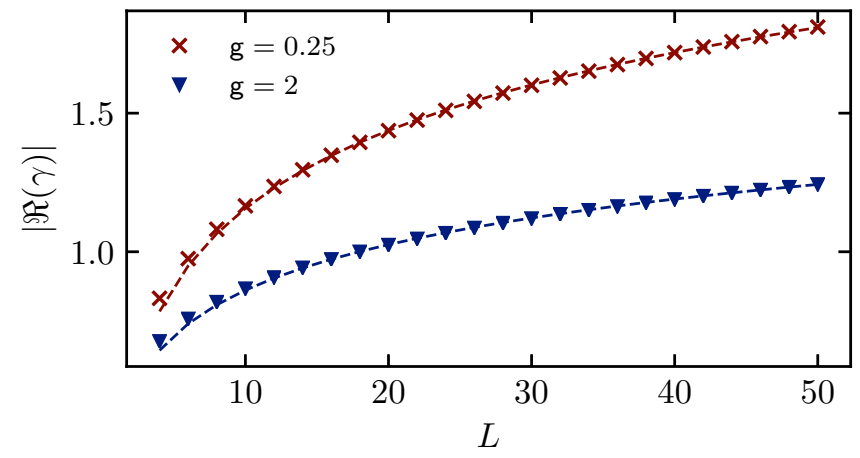

FIG. 4. Absolute value of the real part of the leading eigenvalue for $\omega_{i}=i, i=1 \ldots L$ with fixed $\mathrm{g}$ and varying $L$. Markers indicate exact results; dashed line is a $\log$ arithmic fit $\operatorname{Re}(\gamma) \propto \log L+$ Cst. For $\mathrm{g}=0.25$ the leading eigenvalue is part of a complex conjugate pair, whereas for $\mathrm{g}=2$ the leading eigenvalue is purely real and nondegenerate.

perturbatively. Similarly, we have chosen $\mathrm{g}=2$ to be large enough that the leading eigenvalue is real and proportional to $\mathrm{g}$, but small enough that it is clear that the spectral gap decreases after the exceptional point: for all considered system sizes, the spectral gap at $g=2$ is smaller than the spectral gap at $\mathrm{g}=0.25$. The behavior of the (exact) spectral gap as system size $L$ is varied suggests a logarithmic scaling

$$
\operatorname{Re}(\gamma) \propto \log L+\text { Cst },
$$

and hence only a slow growth as the system size is increased. Comparing the exact results with a logarithmic fit in Fig. 4, the correspondence is excellent for $L \geqslant 10$ until the maximal calculated value $L=50$. The Bethe ansatz allowed us to obtain exact results for a system of $L=50$ spins, where the full Hilbert space has dimension $3^{50} \approx 7.2 \times 10^{23}$.

Moving to the other symmetry sectors, we observe that the eigenvalues are approximately situated on straight lines where $\operatorname{gIm}(\gamma) / \operatorname{Re}(\gamma)= \pm 1 /(2 n+1), n \in \mathbb{Z}$. These can be seen as a remnant from the homogeneous limit, where we already noted that such a quantization occurs. Using both the Bethe states and Bethe equations, we can now generalize this behavior to general inhomogeneous models.

The rapidities solving the Bethe equations (7) can always be subdivided into two classes: the rapidities that are on the same order of magnitude as the $\omega_{j}$, and the rapidities that are large compared to all $\omega_{j}$. Assuming there are $p$ such large rapidities, we can denote the former as $v_{a}, a=1 \ldots q$, and the latter as $w_{a}, a=1 \ldots p$, with $N=p+q$. The states with $p$ large rapidities roughly correspond to the states with $M=$ $-S+p$ in the homogeneous limit, since plugging in the large rapidities in the Bethe state (6) results in generalized raising operators of the form

$$
\sum_{j=1}^{L} \frac{\sqrt{\omega_{j}}}{\omega_{j}-w_{a}} \mathrm{~S}_{j}^{+} \approx-\frac{1}{w_{a}} \sum_{j=1}^{L} \sqrt{\omega_{j}} \mathrm{~S}_{j}^{+},
$$

which reduces to a total spin raising operator in the homogeneous limit where $\omega_{j}=\omega, \forall j$. 
The Bethe equations (7) for these large rapidities can similarly be approximated as

$$
-\frac{\mathrm{g}+i}{2 \mathrm{~g}}-\frac{1}{w_{a}}\left(\sum_{k=1}^{L} \omega_{k}-\sum_{c=1}^{q} v_{c}\right)-\sum_{b \neq a}^{p} \frac{w_{b}}{w_{b}-w_{a}} \approx 0 .
$$

Multiplying this equation with $w_{a}$ and summing over $a=$ $1 \ldots p$, the antisymmetric term drops out and we find (see also Appendix D) that

$$
\frac{\mathrm{g}+i}{2 \mathrm{~g}} \sum_{a=1}^{p} w_{a} \approx p\left(\sum_{c=1}^{q} v_{c}-\sum_{k=1}^{L} \omega_{k}\right)
$$

Plugging this in the expression for the eigenvalue of $\mathcal{L}$ [Eq. (8)], we find that the total eigenvalue $\gamma$ can be written as

$$
\gamma \approx-[(2 p+1) \mathrm{g}+i]\left(\sum_{c=1}^{q} v_{c}-\sum_{k=1}^{L} \omega_{k}\right) .
$$

In the Bethe equations for the remaning "finite" rapidities the dependence on $w_{1}, \ldots, w_{p}$ simply drops out. In the strongcoupling limit these equations are approximately the Bethe equations for the Hermitian model, and finite rapidities can be treated as perturbative solutions to the Bethe equations for the Hermitian model (which is not possible for large rapidities). Furthermore, in the Hermitian model the rapidities only appear as real or as part of a complex conjugate pair, such that their sum is always real-the Hermitian model necessarily has real eigenvalues. In this way we recover the previously observed quantization: for eigenstates of the Liouvillian (5) that can be approximately written as

$$
\left(\sum_{j=1}^{L} \sqrt{\omega_{j}} \mathrm{~S}_{j}^{+}\right)^{p}\left|v_{1} \ldots v_{q}\right\rangle
$$

the eigenvalues satisfy $\operatorname{gIm}(\gamma) / \operatorname{Re}(\gamma) \approx 1 /(2 p+1)$. For these modes, the decay rate is approximately proportional to the oscillation frequency.

\section{CONSERVED CHARGES}

In practice, Bethe equations of the form (7) are rarely solved directly since they are plagued by singularities [49]. Rather, it is always possible to find a set of operator identities for the conserved charges of the integrable model, and these identities can be directly solved to obtain the eigenvalues of the conserved quantities and the integrable model.

While the interpretation of the conserved charges is partly lost in the non-Hermitian case, this formalism can be directly extended to the current case. The Liouvillian still belongs to an extensive set of mutually commuting operators $\left[\mathcal{L}, Q_{j}\right]=$ $0, \forall j$ and $\left[\mathrm{Q}_{j}, \mathrm{Q}_{k}\right]=0, \forall j, k$, defined as

$$
\begin{aligned}
Q_{j}= & i S_{j}^{z}+g\left(S_{j}^{z}\right)^{2}-2 g \sum_{k \neq j}^{L} \frac{\omega_{k}}{\omega_{j}-\omega_{k}} S_{j}^{z} S_{k}^{z} \\
& -2 g \sum_{k \neq j}^{L} \frac{\sqrt{\omega_{j} \omega_{k}}}{\omega_{j}-\omega_{k}}\left(S_{j}^{x} S_{k}^{x}+S_{j}^{y} S_{k}^{y}\right) .
\end{aligned}
$$

These are again a direct extension of the conserved quantities in the Hermitian model, as outlined in Appendix A, and are clearly non-Hermitian. Rather, these commuting quantities exhibit the same pseudo-Hermiticity $/ \mathcal{P} \mathcal{T}$ symmetry of the Liouvillian. The Bethe ansatz states are common eigenstates of all $\mathrm{Q}_{j}$, and the corresponding eigenvalues $\mathrm{q}_{j}$ can be expressed in terms of the rapidities as

$$
\mathbf{q}_{j}=(\mathrm{g}-\mathrm{i})-2 \mathrm{~g} \sum_{a=1}^{N} \frac{v_{a}}{\omega_{j}-v_{a}}+2 \mathrm{~g} \sum_{k \neq j}^{L} \frac{\omega_{k}}{\omega_{j}-\omega_{k}},
$$

where we have made the dependence on the rapidities implicit. Note that $\mathcal{L}$ is not linearly independent of these operators, since

$$
\begin{aligned}
\sum_{j=1}^{L} \omega_{j} \mathrm{Q}_{j}= & i \sum_{j} \omega_{j} \mathrm{~S}_{j}^{z}+\mathrm{g} \sum_{j} \omega_{j}\left(\mathrm{~S}_{j}^{z}\right)^{2} \\
& -\mathrm{g} \sum_{j} \sum_{k \neq j} \sqrt{\omega_{j} \omega_{k}}\left(\mathrm{~S}_{j}^{x} \mathrm{~S}_{k}^{x}+\mathrm{S}_{j}^{y} \mathrm{~S}_{k}^{y}\right) \\
= & \mathcal{L}+\mathrm{g} \sum_{j} \omega_{j}\left[\left(\mathrm{~S}_{j}^{x}\right)^{2}+\left(\mathrm{S}_{j}^{y}\right)^{2}+\left(\mathrm{S}_{j}^{z}\right)^{2}\right] .
\end{aligned}
$$

The final expression has an additional contribution from the Casimir operators of the spin-1 operators, which can be treated as a constant. The same relation holds for the eigenvalues of $\mathcal{L}$ and $Q_{j}$, since all operators mutually commute, and we find that the eigenvalues $\gamma$ of $\mathcal{L}$ can be expanded as

$$
\gamma=\sum_{j=1}^{L} \omega_{j}\left(\mathrm{q}_{j}-2 \mathrm{~g}\right) .
$$

We can similarly recover the conservation of total $\mathrm{S}^{z}=$ $\sum_{j} \mathrm{~S}_{j}^{z}$ from

$$
\sum_{j=1}^{L} \mathrm{Q}_{j}=i \mathrm{~S}^{z}+\mathrm{g}\left(\mathrm{S}^{z}\right)^{2}
$$

Any set of rapidities solving the Bethe equations (7) determines a single Bethe state (6), and this state will be a common eigenstate of all conserved charges with eigenvalues $\left\{q_{1}, q_{2} \ldots q_{L}\right\}$. Rather than first solving the Bethe equations for the rapidities, it is now possible to find a set of equations directly returning this set of eigenvalues, avoiding the explicit use of rapidities. This approach has the advantage that the equations that need to be solved do not display the singular behavior of the regular Bethe equations (7). These equations can be derived using the approach from Ref. [50] and are explicitly derived in Appendix C. Defining shifted eigenvalues

$$
\gamma_{j}=\mathrm{q}_{j}+\mathrm{g}+2 \mathrm{~g} \sum_{k \neq j}^{L} \frac{\omega_{j}}{\omega_{j}-\omega_{k}},
$$

these satisfy the set of equations

$$
\begin{gathered}
{\left[\gamma_{j}-2 \mathrm{~g} \sum_{k \neq j}^{L} \frac{\omega_{j}}{\omega_{j}-\omega_{k}}\right]\left[\gamma_{j}^{2}+\beta^{2}-4 \mathrm{~g} \omega_{j} \sum_{k \neq j}^{L} \frac{\gamma_{j}-\gamma_{k}}{\omega_{j}-\omega_{k}}\right]} \\
=\mathrm{g}\left(\gamma_{j}^{2}+\beta^{2}\right)-4 \mathrm{~g}^{2} \omega_{j}^{2} \sum_{k \neq j}^{L} \frac{\gamma_{j}-\gamma_{k}}{\left(\omega_{j}-\omega_{k}\right)^{2}}
\end{gathered}
$$


with $\beta=1+2 i g(L-N)$. Taking the complex conjugate of these equations maps $\beta \rightarrow \beta^{*}$, and $\beta$ is purely real if $\mathbf{S}^{z}=0$ and hence $N=L$, reflecting the pseudo-Hermiticity in this sector. For $S^{z} \neq 0$ the imaginary part gets mapped to $L-$ $N \rightarrow N-L=L-(2 L-N)$, connecting the sectors with $N$ and $2 L-N$ spin excitations and hence opposite values of $\mathrm{S}^{z}$.

Since these hold for all eigenvalues and all considered operators can be simultaneously diagonalized away from the exceptional points, the operators themselves satisfy the same set of coupled cubic equations away from the exceptional points. Furthermore, since the operators themselves vary continuously at the exceptional points, such operator identities then hold at all values of $\mathrm{g}$ even if the operators are not diagonalizable. These equations in fact form the backbone of our numerical approach. For $\mathrm{g}=0$ the equations decouple and we find $\gamma_{j}\left(\gamma_{j}^{2}+1\right)=0, \forall j$, which can be solved as $\gamma_{j}=0$ or $\gamma_{j}= \pm i$, returning the expected eigenvalues of $i \mathrm{~S}_{j}^{z}$. Solutions at nonzero $\mathrm{g}$ can be obtained by slowly increasing $g$ to its final value and iteratively solving the equations at intermediate values of $\mathrm{g}$ using the solutions at smaller $\mathrm{g}$ as a starting point. In this way the solutions of Eq. (28) can be directly connected to the occupation numbers in the noninteracting limit, providing a way of targeting specific states (see, e.g., Ref. [50] for details). Such an approach is common in Richardson-Gaudin models [36,50-58]. Once these eigenvalues are known, the rapidities can either be extracted from Eq. (23) or the states themselves can be directly expressed in terms of these eigenvalues [54,57]. This equivalence can also be seen as a version of the generalized eigenstate thermalization hypothesis $[59,60]$, since all Bethe states are fully determined by the associated conservation laws.

In terms of the non-Hermitian model considered so far, this has two important consequences. First, we note that the pseudo-Hermiticity-breaking transition can be observed not just in the eigenspectrum of $\mathcal{L}$, but also in the spectrum of all $Q_{j}$. This is illustrated in Fig. 5 for a pair of representative eigenstates. At the transition, the corresponding eigenvalues of all commuting operators change from complex conjugate to purely real. Second, since the states are completely determined by this set of eigenvalues and these eigenvalues are identical at the transition, the states themselves are identical and coalesce: the transition is accompanied by an exceptional point and not an accidental degeneracy.

\section{CONCLUSION}

We discussed the exact solution of a Liouvillian with collective dissipation through a mapping to a non-Hermitian Richardson-Gaudin model. The resulting Hamiltonian is pseudo-Hermitian $/ \mathcal{P} \mathcal{T}$ symmetric, and as the coupling to the environment is increased the eigenvalues change from complex conjugate pairs to purely real. Such a transition is accompanied by a dissipative phase transition and an exceptional point in the spectrum of the Liouvillian, reminiscent of the quantum phase transition in the corresponding Hermitian model. In this way we find an exactly solvable model for an open and interacting quantum system exhibiting nontrivial dynamics.

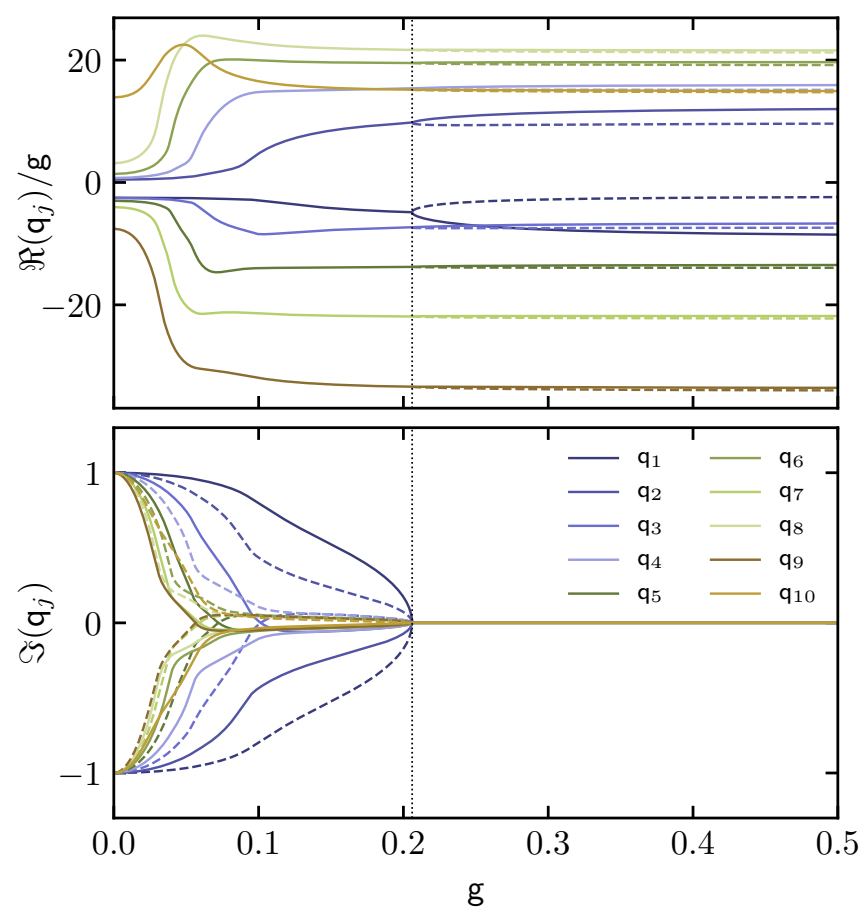

FIG. 5. Real and imaginary parts of the eigenvalues $\mathbf{q}_{j}$ of the commuting operators $\mathrm{Q}_{j}$ with $\omega_{j}=j, j=1 \ldots L$ for $L=10$ and varying $\mathrm{g}$. Full lines indicate the state that is adiabatically connected to the leading mode in the strong-coupling limit, dashed lines indicate the state that is related to this state by $\mathcal{P} \mathcal{T}$ transformation. The transition is marked by a vertical dotted line, and before the transition the eigenvalues are related by complex conjugation, while after the transition the eigenvalues are purely real and no longer related. Note that the real part is rescaled by $\mathrm{g}$.

As discussed in Ref. [27], the balancing of gain and loss is necessary for the mapping to return a solvable Hamiltonian, and it is also a requirement for $\mathcal{P} \mathcal{T}$ symmetry. We have no reason to believe that the model remains integrable once this symmetry is broken, such that integrability here seems to be accompanied by $\mathcal{P} \mathcal{T}$ symmetry. While this work focused on a system of spin- $1 / 2$ degrees of freedom, resulting in a Liouvillian acting trivially on singlet states and nontrivially on triplet states, the construction readily extends to arbitrary spin- $s$ degrees of freedom, leading to a Liouvillian with spin $0,1, \ldots, 2 s$ operators [27].

In the limit where the model is fully homogeneous the eigenspectrum can be expressed in terms of total spin quantum numbers and supports a nontrivial steady state. Away from this limit the decay rates can be analyzed using the exact Bethe ansatz solution, where we find that the nontrivial steady state now decays with a decay rate that increases slowly (logarithmically) with system size. The Bethe ansatz approach is crucial in establishing the logarithmic scaling, since exact eigenvalues can be obtained for system sizes where the Hilbert space is too large for traditional exact methods. For higher excited states in different symmetry sectors we observe that the decay rate is proportional to the oscillation frequency with a quantized prefactor, which is reflected in the eigenvalues organizing themselves in approximately straight lines in the complex plane. 


\section{ACKNOWLEDGMENTS}

We gratefully acknowledge support from EPSRC Grant No. EP/P034616/1. We thank Jan Behrends for useful discussions.

\section{APPENDIX A: HERMITIAN MODEL}

In this Appendix we provide an overview of the exact solution of the Hermitian XXZ Richardson-Gaudin model (see, e.g., [31-36,55,61-63]). For a set of $L$ spin-1 particles, the conserved quantities $Q_{j}, j=1 \ldots L$ are given by

$Q_{j}=S_{j}^{z}+g \sum_{k \neq j}^{L}\left[\frac{\sqrt{\omega_{j} \omega_{k}}}{\omega_{j}-\omega_{k}}\left(S_{j}^{+} S_{k}^{-}+S_{j}^{-} S_{k}^{+}\right)+\frac{\omega_{j}+\omega_{k}}{\omega_{j}-\omega_{k}} S_{j}^{z} S_{k}^{z}\right]$,

satisfying $\left[Q_{j}, Q_{k}\right]=0, \forall j, k$. The Bethe states are defined as

$$
\left|v_{1} \ldots v_{N}\right\rangle=\prod_{a=1}^{N}\left(\sum_{j=1}^{L} \frac{\sqrt{\omega_{j}}}{\omega_{j}-v_{a}} S_{j}^{+}\right)|\emptyset\rangle,
$$

with $|\emptyset\rangle=|1,-1\rangle_{1} \otimes \cdots \otimes|1,-1\rangle_{L}$, and the Bethe equations are given by

$$
\frac{1}{g}+\sum_{j=1}^{L} \frac{\omega_{j}+v_{a}}{\omega_{j}-v_{a}}-\sum_{b \neq a}^{N} \frac{v_{b}+v_{a}}{v_{b}-v_{a}}=0, \quad a=1 \ldots N,
$$

with corresponding eigenvalues

$$
q_{j}=-\left[1+g \sum_{a=1}^{N} \frac{\omega_{j}+v_{a}}{\omega_{j}-v_{a}}-g \sum_{k \neq j}^{L} \frac{\omega_{j}+\omega_{k}}{\omega_{j}-\omega_{k}}\right] .
$$

A Hamiltonian with all-to-all interactions can be constructed by writing

$$
\begin{aligned}
\sum_{j} \omega_{j}\left(Q_{j}+g \mathcal{C}_{j}\right)= & \sum_{j} \omega_{j} S_{j}^{z}\left(1+g \sum_{k} S_{k}^{z}\right) \\
& +g \sum_{j, k} \sqrt{\omega_{j} \omega_{k}}\left(S_{j}^{x} S_{k}^{x}+S_{j}^{y} S_{k}^{y}\right),
\end{aligned}
$$

where $\mathcal{C}_{j}=\left(S_{j}^{x}\right)^{2}+\left(S_{j}^{y}\right)^{2}+\left(S_{j}^{z}\right)^{2}=2$ is the Casimir operator for each algebra. Note that part of the interaction has been absorbed in the first term, but since total spin- $z$ projection is a conserved quantity it can be replaced by its eigenvalue. We can define a new interaction strength

$$
G^{-1}=g^{-1}+\sum_{j} S_{j}^{z}=g^{-1}+(N-L),
$$

and we obtain the Hermitian Hamiltonian from the main text as

$$
\begin{aligned}
& \sum_{i} \omega_{j}\left(Q_{j}+g \mathcal{C}_{j}\right) /\left(1+g \sum_{k} S_{k}^{z}\right) \\
& \quad=\sum_{j} \omega_{j} S_{j}^{z}+G \sum_{j, k} \sqrt{\omega_{j} \omega_{k}}\left(S_{j}^{x} S_{k}^{x}+S_{j}^{y} S_{k}^{y}\right) .
\end{aligned}
$$

The Bethe equations can be rewritten in terms of this new coupling constant as

$$
\frac{1-G}{2 G}+\sum_{j=1}^{L} \frac{\omega_{j}}{\omega_{j}-v_{a}}-\sum_{b \neq a}^{N} \frac{v_{b}}{v_{b}-v_{a}}=0,
$$

as well as the conserved charges

$$
\begin{aligned}
Q_{j} / & \left(1+g \sum_{k} S_{k}^{z}\right) \\
= & S_{j}^{z}-G\left(S_{j}^{z}\right)^{2}+2 G \sum_{k \neq j}^{L}\left[\frac{\sqrt{\omega_{j} \omega_{k}}}{\omega_{j}-\omega_{k}}\left(S_{j}^{x} S_{k}^{x}+S_{j}^{y} S_{k}^{y}\right)\right. \\
& \left.+\frac{\omega_{k}}{\omega_{j}-\omega_{k}} S_{j}^{z} S_{k}^{z}\right]
\end{aligned}
$$

where the corresponding eigenvalues of this rescaled operator can be written as

$$
-(1+G)+2 G \sum_{a=1}^{N} \frac{v_{a}}{\omega_{j}-v_{a}}-2 G \sum_{k \neq j}^{L} \frac{\omega_{k}}{\omega_{j}-\omega_{k}} .
$$

The Liouvillian from the main text now corresponds to a nonHermitian XXZ model with $G=i \mathrm{~g}$.

For completeness, we note that the Hermitian model undergoes a phase transition at $|G|=1$. This can easily be understood since we can write

$$
\begin{aligned}
H & =\sum_{j} \omega_{j} S_{j}^{z}+\frac{G}{2}\left(Q^{\dagger} Q+Q Q^{\dagger}\right) \\
& =\frac{1}{2}\left[Q^{\dagger}, Q\right]+\frac{G}{2}\left(Q^{\dagger} Q+Q Q^{\dagger}\right),
\end{aligned}
$$

with $Q^{\dagger}=\sum_{j} \sqrt{\omega_{j}} S_{j}^{+}$. We can hence rewrite $H$ as

$$
H=\frac{G+1}{2} Q^{\dagger} Q+\frac{G-1}{2} Q Q^{\dagger} .
$$

For $|G|<1$ the ground state is adiabatically connected to the noninteracting ground state at $G=0$, i.e., $\left(S_{1}^{+}\right)^{2}\left(S_{2}^{+}\right)^{2} \ldots\left(S_{N / 2}^{+}\right)^{2}|\emptyset\rangle$ for $\omega_{i}<\omega_{j}$ if $i<j$ and $N$ even, filling the vacuum state with $N$ spin excitations. At $|G|=1$ there is a quantum phase transition for either $N \leqslant L$ (at $G=1$ ) or $N \geqslant L$ (at $G=-1$ ). At the transition the Hamiltonian is positive semidefinite, and the ground state has zero energy and is highly degenerate. For example, for $G=1$ the Hamiltonian can be written as $Q^{\dagger} Q$, for which the ground states are the states annihilated by $Q$, also known as dark states [64], and there is a combinatorial number of such states for $N \leqslant L$. For $G>1$ we find that the ground state is adiabatically connected to $\left(S_{1}^{+}\right)^{2}\left(S_{3}^{+}\right)^{2}\left(S_{5}^{+}\right)^{2} \ldots|\emptyset\rangle$. This transition can be interpreted as a transition to a collective phase, where the ground state reduces to a fully isotropic singlet state $S_{\text {tot }}=0$ in the homogeneous limit.

\section{APPENDIX B: EIGENSPECTRUM FOR $\mathbf{S}^{z} \neq 0$}

For completeness, we show the eigenspectrum of the non-Hermitian Hamiltonian with $S^{z} \neq 0$ in Fig. 6. All eigenvalues remain complex as $g$ is increased, and at large 


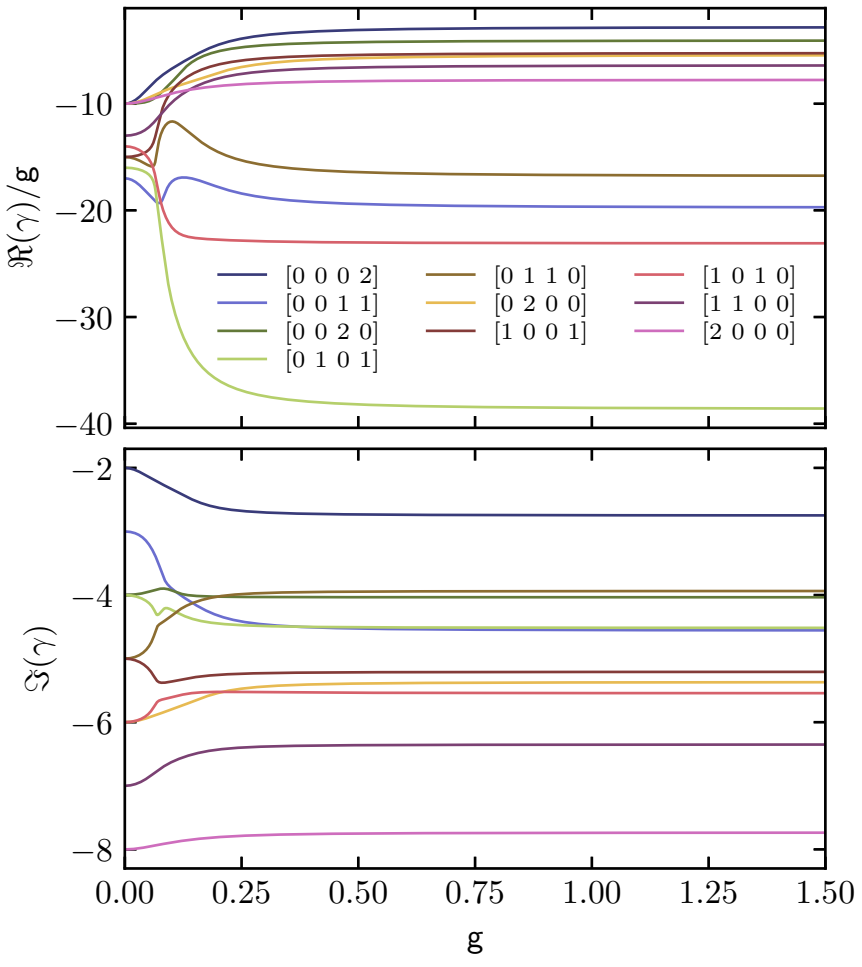

FIG. 6. Eigenspectrum for $L=4$ and $N=2$ with $\omega_{i}=i, \quad i=$ $1 \ldots L$ as $g$ is varied. Note that the real part is rescaled by $g$. The eigenstates are labeled by the spin occupation numbers $\left[n_{1} n_{2} n_{3} n_{4}\right]$ in the noninteracting limit $\mathrm{g}=0$, with $N=\sum_{j} n_{j}$.

coupling strengths the imaginary part is approximately constant whereas the real part is proportional to $\mathrm{g}$.

\section{APPENDIX C: EIGENVALUES OF THE COMMUTING OPERATORS}

In order to obtain the equations determining the eigenvalues of the commuting operators, we define a continuous function

$$
\Lambda(u)=\sum_{a=1}^{N} \frac{1}{u-v_{a}}
$$

following Refs. [50,53]. We again start from the Hermitian model, for which the Bethe equations can be rewritten as

$$
\frac{\alpha}{2 v_{a}}+\sum_{j=1}^{L} \frac{1}{\omega_{j}-v_{a}}-\sum_{b \neq a}^{N} \frac{1}{v_{b}-v_{a}}=0,
$$

where we have introduced $\alpha=1+G^{-1}+2(L-N)$ for convenience. We then find

$$
\begin{aligned}
& {[\Lambda(u)]^{2}} \\
& \quad=\sum_{a} \frac{1}{\left(u-v_{a}\right)^{2}}+\sum_{a} \sum_{b \neq a} \frac{1}{\left(u-v_{a}\right)} \frac{1}{\left(u-v_{b}\right)} \\
& \quad=\sum_{a} \frac{1}{\left(u-v_{a}\right)^{2}}+2 \sum_{a} \sum_{b \neq a} \frac{1}{\left(u-v_{a}\right)} \frac{1}{\left(v_{a}-v_{b}\right)}
\end{aligned}
$$

$$
\begin{aligned}
= & \sum_{a} \frac{1}{\left(u-v_{a}\right)^{2}}-2 \sum_{a} \frac{1}{\left(u-v_{a}\right)}\left[\frac{\alpha}{2 v_{a}}+\sum_{j} \frac{1}{\omega_{j}-v_{a}}\right] \\
= & \sum_{a} \frac{1}{\left(u-v_{a}\right)^{2}}-\frac{\alpha}{u} \sum_{a}\left[\frac{1}{u-v_{a}}+\frac{1}{v_{a}}\right] \\
& -2 \sum_{j \neq i} \sum_{a} \frac{1}{\omega_{j}-u}\left[\frac{1}{u-v_{a}}-\frac{1}{\omega_{j}-v_{a}}\right] \\
& -2 \sum_{a} \frac{1}{\left(u-v_{a}\right)\left(\omega_{i}-v_{a}\right)},
\end{aligned}
$$

where we have performed a partial fraction decomposition in the second and fourth lines, assuming $u \neq \omega_{j}, j \neq i$ for a fixed $i$, and used the Bethe equations to evaluate the summation $b \neq a$. These can be further evaluated since

$$
\sum_{a} \frac{\alpha}{2 v_{a}}=-\sum_{j, a} \frac{1}{\omega_{j}-v_{a}}=-\sum_{j} \Lambda\left(\omega_{j}\right),
$$

again making use of the Bethe equations, to find

$$
\begin{aligned}
{[\Lambda(u)]^{2}=} & -\frac{\alpha}{u} \Lambda(u)+\frac{2}{u} \sum_{j} \Lambda\left(\omega_{j}\right)+2 \sum_{j \neq i} \frac{\Lambda(u)-\Lambda\left(\omega_{j}\right)}{u-\omega_{j}} \\
& +\sum_{a} \frac{1}{\left(u-v_{a}\right)^{2}}-2 \sum_{a} \frac{1}{\left(u-v_{a}\right)\left(\omega_{i}-v_{a}\right)} .
\end{aligned}
$$

We can plug in $u=\omega_{i}$ and multiply the equation with $\omega_{i}$ to find

$$
\begin{aligned}
\omega_{i} \Lambda\left(\omega_{i}\right)^{2}= & -\alpha \Lambda\left(\omega_{i}\right)+2 \sum_{j} \Lambda\left(\omega_{j}\right)-\sum_{a} \frac{\omega_{i}}{\left(\omega_{i}-v_{a}\right)^{2}} \\
& +2 \omega_{i} \sum_{j \neq i} \frac{\Lambda\left(\omega_{i}\right)-\Lambda\left(\omega_{j}\right)}{\omega_{i}-\omega_{j}} .
\end{aligned}
$$

This is almost a closed set of equations for $\left\{\Lambda\left(\omega_{1}\right) \ldots \Lambda\left(\omega_{L}\right)\right\}$, except for the dependence on $\sum_{a} 1 /\left(\omega_{i}-v_{a}\right)^{2}=-\Lambda^{\prime}\left(\omega_{i}\right)$. This dependence can be removed by taking the derivative of the above equations with respect to $u$ and again evaluating at $u=\omega_{i}$, leading to

$$
\begin{aligned}
& 2 \Lambda\left(\omega_{i}\right) \Lambda^{\prime}\left(\omega_{i}\right) \\
& =-\frac{\alpha}{\omega_{i}} \Lambda^{\prime}\left(\omega_{i}\right)+\frac{\alpha-2}{\omega_{i}^{2}} \Lambda\left(\omega_{i}\right)-\frac{2}{\omega_{i}^{2}} \sum_{j \neq i} \Lambda\left(\omega_{j}\right) \\
& \quad+2 \sum_{j \neq i} \frac{\Lambda^{\prime}\left(\omega_{i}\right)}{\omega_{i}-\omega_{j}}-2 \sum_{j \neq i} \frac{\Lambda\left(\omega_{i}\right)-\Lambda\left(\omega_{j}\right)}{\left(\omega_{i}-\omega_{j}\right)^{2}} .
\end{aligned}
$$

This equation can be used to express $\Lambda^{\prime}\left(\omega_{i}\right)$ in terms of $\left\{\Lambda\left(\omega_{1}\right) \ldots \Lambda\left(\omega_{L}\right)\right\}$, which can then be plugged into the previously obtained equations to obtain a closed set of equations. Defining

$$
\gamma_{i}=2 \omega_{i} G \Lambda\left(\omega_{i}\right)+2 G(L-N)+1
$$

then returns Eq. (28) after some straightforward manipulations. 


\section{APPENDIX D: HEINE-STIELTJES CONNECTION}

In this Appendix, we find an explicit expression for the large rapidities $w_{1} \ldots w_{p}$ in terms of the finite rapidities $v_{1} \ldots v_{q}$, given Eq. (18). Defining $\Delta=\sum_{j=1}^{L} \omega_{j}-\sum_{c=1}^{q} v_{c}$, we can write the Bethe equations for the large rapidities as

$$
-\frac{\mathrm{g}+i}{2 \mathrm{~g}}-\frac{\Delta}{w_{a}}-\sum_{b \neq a}^{p} \frac{w_{b}}{w_{b}-w_{a}}=0, \quad a=1 \ldots p .
$$

We can relate the solutions of these equations to the roots of associated Laguerre functions. The associated Laguerre polynomials $L_{n}^{\alpha}(z)$ satisfy the differential equation

$$
z P^{\prime \prime}(z)+(1+\alpha-z) P^{\prime}(z)+n P(z)=0,
$$

for $P(z)=L_{n}^{\alpha}(z)$, and from the Heine-Stieltjes connection $[65,66]$ the roots $z_{a}, a=1 \ldots n$ are coupled through

$$
1-\frac{1+\alpha}{z_{a}}-2 \sum_{b \neq a}^{n} \frac{1}{z_{a}-z_{b}}=0, \quad a=1 \cdots n .
$$

The Bethe equations for the large roots can be recast in terms of $x_{a}=1 / w_{a}$ as

$$
-\frac{\mathrm{g}+i}{2 \mathrm{~g}} \frac{1}{x_{a}}-\Delta-\sum_{b \neq a}^{p} \frac{1}{x_{a}-x_{b}}=0 .
$$

These are exactly the equations for the roots of the associated Laguerre polynomials $L_{n}^{\alpha}(z)$ with $z_{a}=-2 \Delta x_{a}=-2 \Delta / w_{a}$, $n=p$, and $\alpha=i / \mathrm{g}$. We can also recover the sum rule from the main text since

$$
\sum_{a=1}^{n} \frac{1}{z_{a}}=-\frac{P^{\prime}(0)}{P(0)}=\frac{n}{1+\alpha},
$$

which here reduces to

$$
\sum_{a=1}^{q} w_{a}=-2 \Delta \frac{p}{1+i / g}
$$

[1] H.-P. Breuer and F. Petruccione, The Theory of Open Quantum Systems (Oxford University Press, New York, 2007).

[2] A. Mostafazadeh, Pseudo-hermitian representation of quantum mechanics, Int. J. Geom. Methods Mod. Phys. 07, 1191 (2010).

[3] R. El-Ganainy, K. G. Makris, M. Khajavikhan, Z. H. Musslimani, S. Rotter, and D. N. Christodoulides, NonHermitian physics and PT symmetry, Nat. Phys. 14, 11 (2018).

[4] J. Huber, P. Kirton, S. Rotter, and P. Rabl, Emergence of PTsymmetry breaking in open quantum systems, Sci. Post. Phys. 9, 052 (2020).

[5] Y. Nakanishi and T. Sasamoto, PT phase transition in open quantum systems with Lindblad dynamics, arXiv:2104.07349.

[6] W. D. Heiss, The physics of exceptional points, J. Phys. A: Math. Theor. 45, 444016 (2012).

[7] Y. Ashida, Z. Gong, and M. Ueda, Non-Hermitian physics, Adv. Phys. 69, 249 (2020).

[8] E. J. Bergholtz, J. C. Budich, and F. K. Kunst, Exceptional topology of non-Hermitian systems, Rev. Mod. Phys. 93, 015005 (2021).

[9] T. Prosen, Third quantization: A general method to solve master equations for quadratic open Fermi systems, New J. Phys. 10, 043026 (2008).

[10] T. Prosen and B. Žunkovič, Exact solution of Markovian master equations for quadratic Fermi systems: Thermal baths, open XY spin chains and non-equilibrium phase transition, New J. Phys. 12, 025016 (2010).

[11] M. van Caspel, S. E. Tapias Arze, and I. Pérez Castillo, Dynamical signatures of topological order in the driven-dissipative Kitaev chain, Sci. Post. Phys. 6, 026 (2019).

[12] P. L. Krapivsky, K. Mallick, and D. Sels, Free fermions with a localized source, J. Stat. Mech. (2019) 113108.

[13] N. Shibata and H. Katsura, Dissipative quantum Ising chain as a non-hermitian ashkin-teller model, Phys. Rev. B 99, 224432 (2019).

[14] E. Vernier, Mixing times and cutoffs in open quadratic fermionic systems, Sci. Post. Phys. 9, 049 (2020).

[15] S. Lieu, M. McGinley, and N. R. Cooper, Tenfold Way for Quadratic Lindbladians, Phys. Rev. Lett. 124, 040401 (2020).
[16] V. Alba and F. Carollo, Noninteracting fermionic systems with localized dissipation: Exact results in the hydrodynamic limit, arXiv:2103.05671.

[17] T. Prosen, Open XXZ Spin Chain: Nonequilibrium Steady State and a Strict Bound on Ballistic Transport, Phys. Rev. Lett. 106, 217206 (2011).

[18] T. Prosen, Exact Nonequilibrium Steady State of a Strongly Driven Open XXZ Chain, Phys. Rev. Lett. 107, 137201 (2011).

[19] D. Karevski, V. Popkov, and G. M. Schütz, Exact Matrix Product Solution for the Boundary-Driven Lindblad XXZ Chain, Phys. Rev. Lett. 110, 047201 (2013).

[20] E. Ilievski, Dissipation-driven integrable fermionic systems: From graded Yangians to exact nonequilibrium steady states, Sci. Post. Phys. 3, 031 (2017).

[21] M. Vanicat, L. Zadnik, and T. Prosen, Integrable Trotterization: Local Conservation Laws and Boundary Driving, Phys. Rev. Lett. 121, 030606 (2018).

[22] G. T. Landi, D. Poletti, and G. Schaller, Non-equilibrium boundary driven quantum systems: Models, methods and properties, arXiv:2104.14350.

[23] M. V. Medvedyeva, F. H. L. Essler, and T. Prosen, Exact Bethe Ansatz Spectrum of a Tight-Binding Chain with Dephasing Noise, Phys. Rev. Lett. 117, 137202 (2016).

[24] A. A. Ziolkowska and F. Essler, Yang-Baxter integrable Lindblad equations, Sci. Post Phys. 8, 044 (2020).

[25] B. Buča, C. Booker, M. Medenjak, and D. Jaksch, Bethe ansatz approach for dissipation: Exact solutions of quantum manybody dynamics under loss, New J. Phys. 22, 123040 (2020).

[26] M. de Leeuw, C. Paletta, and B. Pozsgay, Constructing Integrable Lindblad Superoperators, Phys. Rev. Lett. 126, 240403 (2021).

[27] D. A. Rowlands and A. Lamacraft, Noisy Spins and the Richardson-Gaudin Model, Phys. Rev. Lett. 120, 090401 (2018).

[28] P. Ribeiro and T. Prosen, Integrable Quantum Dynamics of Open Collective Spin Models, Phys. Rev. Lett. 122, 010401 (2019). 
[29] S. Lerma-Hernández, A. Rubio-García, and J. Dukelsky, Trigonometric $S U(N)$ Richardson-Gaudin models and dissipative multi-level atomic systems, J. Phys. A: Math. Theor. 53, 395302 (2020).

[30] Á. Rubio-García, R. A. Molina, and J. Dukelsky, From integrability to chaos in quantum Liouvillians, arXiv:2102.13452.

[31] G. Ortiz, R. Somma, J. Dukelsky, and S. Rombouts, Exactlysolvable models derived from a generalized Gaudin algebra, Nucl. Phys. B 707, 421 (2005).

[32] M. Ibañez, J. Links, G. Sierra, and S.-Y. Zhao, Exactly solvable pairing model for superconductors with $p_{x}+i p_{y}$-wave symmetry, Phys. Rev. B 79, 180501(R) (2009).

[33] S. M. A. Rombouts, J. Dukelsky, and G. Ortiz, Quantum phase diagram of the integrable $p_{x}+i p_{y}$ fermionic superfluid, Phys. Rev. B 82, 224510 (2010).

[34] M. Van Raemdonck, S. De Baerdemacker, and D. Van Neck, Exact solution of the $p_{x}+i p_{y}$ pairing Hamiltonian by deforming the pairing algebra, Phys. Rev. B 89, 155136 (2014).

[35] J. Links, I. Marquette, and A. Moghaddam, Exact solution of the $p+i p$ Hamiltonian revisited: Duality relations in the holepair picture, J. Phys. A: Math. Theor. 48, 374001 (2015).

[36] P. W. Claeys, S. De Baerdemacker, and D. Van Neck, ReadGreen resonances in a topological superconductor coupled to a bath, Phys. Rev. B 93, 220503(R) (2016).

[37] T. E. Lee, S. Gopalakrishnan, and M. D. Lukin, Unconventional Magnetism via Optical Pumping of Interacting Spin Systems, Phys. Rev. Lett. 110, 257204 (2013).

[38] J. Jin, A. Biella, O. Viyuela, L. Mazza, J. Keeling, R. Fazio, and D. Rossini, Cluster Mean-Field Approach to the Steady-State Phase Diagram of Dissipative Spin Systems, Phys. Rev. X 6, 031011 (2016).

[39] W. Casteels, R. M. Wilson, and M. Wouters, Gutzwiller Monte Carlo approach for a critical dissipative spin model, Phys. Rev. A 97, 062107 (2018).

[40] A. Biella, J. Jin, O. Viyuela, C. Ciuti, R. Fazio, and D. Rossini, Linked cluster expansions for open quantum systems on a lattice, Phys. Rev. B 97, 035103 (2018).

[41] D. Huybrechts, F. Minganti, F. Nori, M. Wouters, and N. Shammah, Validity of mean-field theory in a dissipative critical system: Liouvillian gap, $\mathbb{P T}$-symmetric antigap, and permutational symmetry in the $X Y Z$ model, Phys. Rev. B 101, 214302 (2020).

[42] A. Mostafazadeh, Pseudo-Hermiticity versus PT symmetry: The necessary condition for the reality of the spectrum of a non-Hermitian Hamiltonian, J. Math. Phys. 43, 205 (2002).

[43] Technically, this operator generates the evolution of the corresponding correlation functions, and its Hermitian conjugate generates the evolution of the operators. We write the Liouvillian in this way in order to make the connection with the literature.

[44] C. M. Bender and S. Boettcher, Real Spectra in Non-Hermitian Hamiltonians Having $\mathcal{P} \mathcal{T}$ Symmetry, Phys. Rev. Lett. 80, 5243 (1998).

[45] T. Prosen, PT-Symmetric Quantum Liouvillean Dynamics, Phys. Rev. Lett. 109, 090404 (2012).

[46] J. G. Hirsch, A. Mariano, J. Dukelsky, and P. Schuck, Fully selfconsistent RPA description of the many level pairing model, Ann. Phys. 296, 187 (2002).
[47] D. L. Andrews and T. Thirunamachandran, On threedimensional rotational averages, J. Chem. Phys. 67, 5026 (1977).

[48] F. R. Bernhart, Catalan, Motzkin, and Riordan numbers, Discrete Mathematics Selected papers in honor of Henry W. Gould, Discrete Math. 204, 73 (1999).

[49] S. De Baerdemacker, Richardson-Gaudin integrability in the contraction limit of the quasispin, Phys. Rev. C 86, 044332 (2012).

[50] P. W. Claeys, S. De Baerdemacker, M. Van Raemdonck, and D. Van Neck, Eigenvalue-based method and form-factor determinant representations for integrable XXZ Richardson-Gaudin models, Phys. Rev. B 91, 155102 (2015).

[51] O. Babelon and D. Talalaev, On the Bethe ansatz for the JaynesCummings-Gaudin model, J. Stat. Mech. (2007) P06013.

[52] A. Faribault, O. El Araby, C. Sträter, and V. Gritsev, Gaudin models solver based on the correspondence between Bethe ansatz and ordinary differential equations, Phys. Rev. B 83, 235124 (2011).

[53] O. El Araby, V. Gritsev, and A. Faribault, Bethe ansatz and ordinary differential equation correspondence for degenerate Gaudin models, Phys. Rev. B 85, 115130 (2012).

[54] P. W. Claeys, S. De Baerdemacker, and D. Van Neck, Inner products in integrable Richardson-Gaudin models, Sci. Post. Phys. 3, 028 (2017).

[55] P. W. Claeys, Richardson-Gaudin models and broken integrability, Ph.D. thesis, Ghent University, 2018.

[56] C. Dimo and A. Faribault, Quadratic operator relations and Bethe equations for spin-1/2 Richardson-Gaudin models, J. Phys. A: Math. Theor. 51, 325202 (2018).

[57] A. Faribault and C. Dimo, "Bethe-Ansatz-free" eigenstates of spin-1/2 Richardson-Gaudin integrable models, arXiv: 1812.06428.

[58] P. W. Claeys, C. Dimo, S. D. Baerdemacker, and A. Faribault, Integrable spin-1/2 Richardson-Gaudin XYZ models in an arbitrary magnetic field, J. Phys. A: Math. Theor. 52, 08LT01 (2019).

[59] L. D’Alessio, Y. Kafri, A. Polkovnikov, and M. Rigol, From quantum chaos and eigenstate thermalization to statistical mechanics and thermodynamics, Adv. Phys. 65, 239 (2016).

[60] E. Ilievski, E. Quinn, and J.-S. Caux, From interacting particles to equilibrium statistical ensembles, Phys. Rev. B 95, 115128 (2017).

[61] T. Skrypnyk, Non-skew-symmetric classical $r$-matrices and integrable cases of the reduced BCS model, J. Phys. A: Math. Theor. 42, 472004 (2009).

[62] T. Skrypnyk, Non-skew-symmetric classical $r$-matrices, algebraic Bethe ansatz, and Bardeen-Cooper-Schrieffer-type integrable systems, J. Math. Phys. 50, 033504 (2009).

[63] T. Skrypnyk, Classical $r$-matrices, "elliptic" BCS and Gaudintype hamiltonians and spectral problem, Nucl. Phys. B 941, 225 (2019).

[64] T. Villazon, A. Chandran, and P. W. Claeys, Integrability and dark states in an anisotropic central spin model, Phys. Rev. Research 2, 032052(R) (2020).

[65] T. J. Stieltjes, Un théorème d'algèbre, Acta Math. 6, 319 (1885).

[66] B. Sriram Shastry and A. Dhar, Solution of a generalized Stieltjes problem, J. Phys. A 34, 6197 (2001). 